\title{
Female Epispadiasis: A Case report
}

\author{
Bhat $\mathbf{N}^{1}$, Wani $\mathbf{I}^{2}$ \\ ${ }^{1}$ Dr. Nisar Bhat, MBBS. MS. MCh, Assistant Professor, Department of Paediatric Surgery. ${ }^{2}$ Dr. Imtiaz Wani. MBBS. MS. \\ Department of Paediatric Surgery. Both from the Sheri-Kashmir Institute of Medical sciences, Srinagar, Kashmir,India.
}

Address for correspondence: Dr. Nisar Bhat, E-mail: nisarabuhade@yahoo.com

\begin{abstract}
Isolated female epispadias without exstrophy is rare to see. It is often overlooked cause of incontinence in a female child. A case of isolated female epispadias without exstrophy in an 11-year-old girl who presented with primary urinary incontinence since birth is reported. She had never had a genitalia examination done by any treating clinician. On examination, she had a bifid clitoris and ill developed labia minora. Voiding cystourethrogram showed small sized bladder with no reflux. Bladder neck reconstruction was done transvesically, with tubularisation of urethra. The case report reflects the importance of physical examination in primary incontinence which can make an early diagnosis and treatment and prevent stigmata of incontinence.
\end{abstract}

\section{Introduction}

Female epispadias is a rare congenital anomaly with an incidence of 1 in 4840, 000 female children ${ }^{1}$. The anomaly is a type of the exstrophy- epispadias complex and is considered a highly morbid condition. Symptoms are primary urinary incontinence and abnormal anatomical features. This female episadias is obvious and easy to diagnose, but less severe variants may still involve urethral sphincter incompetence in the absence of obvious clinical signs. Isolated female epispadias without exstrophy is an extremely rare syndrome ${ }^{2}$. The key diagnostic features include drug-resistant wetting, a very low leak pressure on cystometrography, a palpable gap in the pubic symphysis and an abnormal ovalshaped external urethral meatus. A bifid clitoris is an important sign but not always present. Lack of proper examination of external genitalia often leads to its under-diagnosis and mismanagement. If unrecognized and untreated, there is overwhelming psychosocial and psychosexual problems for the affected patient. Surgery generally leads to the ability to control the flow of urine and a good cosmetic outcome.

\section{The Case}

An 11-year-old female child presented with primary urinary continence or chronic wetting since birth. She was being managed as a case of chronic bed wetting and had never had a perineal examination by the treating clinicians till she was referred to our hospital with incontinence for last 11 years.

Child had an anxious look. General physical examination, systemic including the central nervous system examination was unremarkable. On physical examination, bifid clitoris, depressed mons covered with a smooth, glabrous skin was present(Fig.1). Labia minora were ill developed and terminated anteriorly to corresponding half of bifid clitoris (Fig.2 and 3). The vagina and hymen were normal. On separating the labia, a wide and patulous external urethral meatus was found.Multiple perineal hypopigmentic spots were present due to continous urine dribbling.(Fig.3). All the baseline investigations were : Perineal hypopigmentic spots due to continous urine dribbling normal, Urine examination and culture were sterile. X-ray spine was normal whereas $\mathrm{X}$-ray pelvis showed pubic diastasis. Abdominal pelvic sonography was normal. Voiding cystourethrogram revealed a moderately small capacity urinary bladder, wide bladder neck and dilated urethra in its entire length without vesico ureteric reflux. Cystoscopy confirmed the findings of micturating cystourethrogram.

Surgical intervention in the form of bladder neck reconstruction transvesically, tubularisation of urethra and uerthroplasty was done. Patient had an uneventful postoperative period. On follow up she is completely dry four last 4 years. 


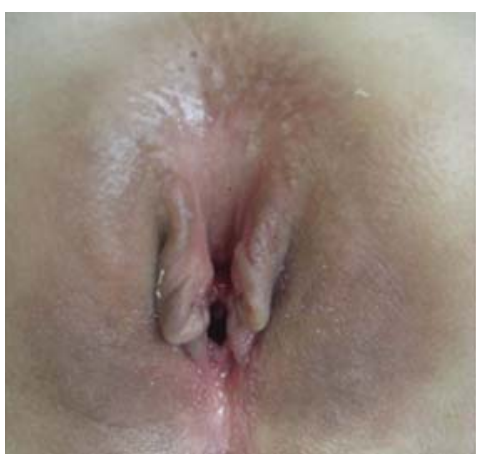

Fig 1: Showing depressed mons covered with a smooth, glabrous skin.

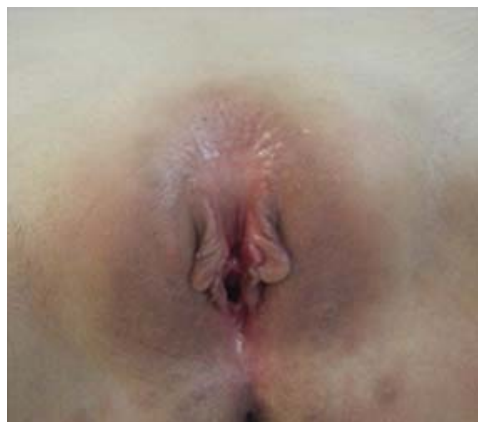

Fig 2: Showing Bifid clitoris with ill defined labia minora.

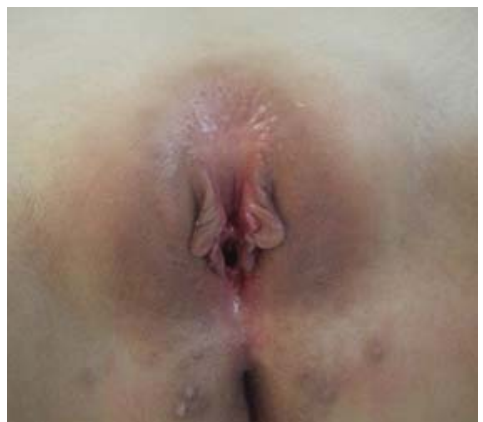

Fig 3: Showing perineal hypopigmentic spots due to continous dribbling of urine.

\section{Discussion}

Epispadias in females is an uncommon congenital anomaly but actually it may be three times more common than primarily expected because the diagnosis is often missed even by the experienced clinicians and patients with lesser degrees of epispadias without incontinence go unnoticed ${ }^{3}$. This condition is a partial form of a spectrum of failures of abdominal and pelvic fusion in the first months of embryogenesis known as the exstrophy -epispadias complex. It occurs as a result of defective migration of the genital tubercle primordi to the cloacal membrane, and so malformation of the genital tubercle, at about the 5th week of gestation. Causes are still essentially unknown, with viral infection during infection proposed as a leading theory; chemical intake of the pregnant mother has been incriminated as a cause. Hormonal evaluation showed functional hyperandrogenism, and peripheral blood arraybased comparative genomic hybridization showed no chromosomal deletions or duplications ${ }^{4}$. While epispadias is inherent in all cases of exstrophy it can also, much less frequently, appear in isolation as the least severe form of the complex spectrum

The characteristic genital defect seen in female episadias is a bifid clitoris, mons depressed covered by a smooth, glabrous skin, labia minora ill developed and terminated anteriorly to corresponding half of bifid clitoris and patulous wide meatus. Davis described that the appearance of external genitalia varies from lesser degree of epispadias with patulous urethral orifice to intermediate type with urethra dorsally split along most of its length to the most severe type which involves the entire length of urethra rendering the sphincter mechanism incompetent ${ }^{5}$ The vagina and internal genital organs are usually normal. The bladder is often small capacity with poorly developed bladder neck and incompetent sphincter mechanism. The ureters often enter laterally with a straighter course so that reflux occurs in around $30-75 \%$ of cases ${ }^{6}$.

Radiological evaluation is required to identify reflux and rule out other causes on in continence Pelvic $\mathrm{x}$-ray ultrasound of the urogenital system, intravenous pyelogram (IVP), and urodynamic studies form important investigation tools.Urodynamic studies help in confirming the poor outlet resistance. Cystoscopy will assess bladder capacity \& the position of ureteric orifices.It is to be stressed that careful examination of the external genitalia by separating labia majora is pivotal in diagnosis of this condition.

Treatment of female epispadias is targeted to achieve urinary continence, preserve upper urinary tract and reconstruction of functionally as well as cosmetically acceptable external genitalia. Reconstructive surgery of the urethra and bladder neck improves the outcome in case of early intervention. Genitoplasty, ureteral reimplantation for vesicoureteric reflux, urethral and bladder neck reconstruction, and augmentation for a small capacity bladder are surgical options available for complete reconstruction in female epispadias. One stage reconstruction involving genital, urethral and bladder neck reconstruction has satisfactory results. Young dees procedure and simultaneous perineal reconstruction has stood the test of time, with continence rate of $60 \%$ to $85.5 \%{ }^{6,7,8}$. However, this requires extensive mobilization and is difficult to achieve continence in a small capacity bladder. Gearhart et $\mathrm{al}^{9}$ advovocated urethral and genital reconstruction in 
children aged 18 months to 2 years and have advocated delaying the bladder neck reconstruction until the child is 4 to 5 years old and achieved continence of $87.5 \%$. A single-stage new technique of perineal urethroplasty with double breasting of the urethra and bladder neck and sphincteroplasty in female patients with epispadias focusing all factors important for continence has advocated in in female epispadias repair ${ }^{10}$.

\section{Conclusion}

The importance of a careful examination in an incontinent female patient should be emphasized to reduce the chances of mismanagement and decreases the morbidity associated with this disease that usually results from the lack of knowledge even among the experienced clinicians as was seen in our patient.

\section{References}

1. Gearhart JP. Exstrophy, epispadias \& other bladder anomalies. In walsh PC, et al (Eds.). Campbell's Urology, 7th ed Philadelphia, W B Saunders, 1998; pp. 2136.

2. Atilgan D, Uluocak N, Erdemir F, Parlaktas B. Female Epispadias: A Case Report and Review of the Literature. Kaohsiung $J$ Med Sci. 2009;25(11):613-616.
3. Allen L, Rodjani A, Kelly J, Maki I, Hutson M. Female Epispadias: are we missing the diagnosis? BJU International 2004;94(4):613-615.

4. Tantibhedhyangkul J, Copland S, Haqq A, Price T. A case of female epispadias Fertility Sterility. 2008;90(5):2017.e1-2017.e3.

5. Davis DM: Epispadias in female \& its surgical treatment, Surg Gynec Obst. 1928;47:680.

6. Kramer SL, Kelalis PP. Assessment of urinary continence in Epispadias review of 94 cases. J Urol 1982;128:290.

7. Tango E.A.Bladder neck reconstruction for total urinary incontinence. J Urol 1981;125:321-326.

8. De-Jong T,Dik P, Klijn A. Female epispadias repair:a new 1-stage technique. J Urol 2000;164:492-494.

9. Gearhart J, Pepps P, D.S, R JeffS .Complete genitourinary re-construction in female epispadias. J Urol 1993;149:1110-1113.

10. Sharma R, Gajendra S, Dejong P et al. SingleStage Perineal Urethroplasty for Continence in Female Epispadias: A Preliminary Report. Urology 2008;72 (2);300-304.

\section{How to cite this article?}

Wani I, Bhat N. Female Epispadiasis: A Case report. J Nepal Paediatr Soc 2011;31(3):244-246. 